CLINICAL STUDY

\title{
Regional fat distribution in adolescents with anorexia nervosa: effect of duration of malnutrition and weight recovery
}

\author{
María Teresa García De Álvaro ${ }^{1}$, María Teresa Muñoz-Calvo ${ }^{1,2}$, Vicente Barrios ${ }^{1,2}$, Guillermo Martínez ${ }^{3}$, \\ Gabriel Ángel Martos-Moreno ${ }^{1,2}$, Federico Hawkins ${ }^{3}$ and Jesús Argente ${ }^{1,2}$ \\ ${ }^{1}$ Department of Endocrinology, Hospital Infantil Universitario Niño Jesús, Universidad Autónoma de Madrid, Avda. Menéndez Pelayo, 65, E-28009 \\ Madrid, Spain, ${ }^{2}$ Ciber Fisiopatología Obesidad y Nutrición (CB06/03) Instituto de Salud Carlos III, E-28009 Madrid, Spain and ${ }^{3}$ Department of \\ Endocrinology, Hospital Universitario 12 de Octubre and Universidad Complutense, E-28041 Madrid, Spain \\ (Correspondence should be addressed to J Argente; Email: argentefen@terra.es)
}

\begin{abstract}
Objective: This study addresses the influence of the duration of malnutrition and the effect of weight recovery on regional fat mass distribution in moderately malnourished adolescents with anorexia nervosa (AN).

Study design: We measured total and regional fat mass and leptin levels in 42 restrictive AN female adolescents and 23 controls. AN patients, followed over 2 years, were divided into three groups: prolonged moderate malnutrition (PM; secondary amenorrhea for over 1 year, $n=14$ ); SM, short period of moderate malnutrition (secondary amenorrhea for less than 1 year, $n=13$ ); and $R$, recovered from AN (BMI, body mass index and menses recovered for over 6 months, $n=15$ ).

Results: Total, trunk, and extremity fat mass were reduced in the PM and SM groups $(P<0.05)$, whereas only PM patients showed altered regional fat distribution with a low trunk to extremity fat ratio $(P<0.05)$. BMI increased after 12 months only in the SM group $(P<0.05)$, with menses resumption in $69 \%$ of these patients and BMI normalization at 24 months. Their regional fat distribution was similar to controls throughout the study. No difference in any parameter was found between the $R$ group and the controls. Conclusion: Prolonged malnutrition, but not weight recovery, is associated with an abnormal regional fat distribution pattern in moderately malnourished $\mathrm{AN}$ adolescents.
\end{abstract}

European Journal of Endocrinology 157 473-479

\section{Introduction}

Anorexia nervosa (AN) is a high prevalence (1) eating disorder characterized by low body weight and severe distortion of body compartments, associated with deficiencies in bone, fat, and lean tissue. Weight loss is mainly due to fat loss, with some authors reporting differences in the regional distribution of this fat mass reduction (2).

Nutritional recovery is the main therapy for patients with AN, but this may result in significant changes in body composition, especially in regional fat mass distribution. Conflicting results regarding a possible trend toward central fat accumulation and trunk adiposity have been reported and suggested that this is influenced by the age of the patients, the degree of fat mass restoration, and the time required for weight recovery $(3,4)$.

Fat tissue is an active player in energy balance and metabolic homeostasis, synthesizing several peptides with endocrine actions. Among these peptides, leptin acts as a satiety signal regulating energy intake and expenditure, and subsequently body weight. Hence, its levels are influenced by body fat mass and distribution (5). In AN subjects, serum leptin levels are dramatically reduced and its physiological regulation altered (6), which could contribute to the pathogenesis of persisting abnormalities observed in these patients.

Most published studies concerning body composition disturbances in AN have been performed in patients with severe malnutrition. However currently, patients with eating disorders are more heterogeneous with different degrees of undernutrition and clinical abnormalities, with most cases being less severe than in past decades. This is at least partially due to an earlier diagnosis of the illness that contributes to better treatment and prognosis of these patients.

The aims of this study were to 1) evaluate the influence of the duration of malnutrition on regional fat mass distribution in $\mathrm{AN}$, and 2) analyze the effect of weight recovery on fat tissue distribution.

\section{Patients and methods}

\section{Patients}

We carried out a prospective follow-up study of 42 female adolescents with restrictive $\mathrm{AN}$, as determined by 
DSM-IV-TR criteria (7), over a 24-month period. The age of the patients ranged between 12.3 and 17.8 years. The patients were referred to the Department of Endocrinology from the Unit of Eating Disorders at the Hospital Niño Jesús in Madrid, Spain. The patients were divided into three groups according to their nutritional status, presence or absence of amenorrhea, and its duration at the beginning of the study. The following groups were studied;

(PM) prolonged moderate malnutrition; $n=14$ : These patients had malnutrition (body mass index (BMI) $-1.69 \pm 0.63$ s.D.s) and secondary amenorrhea for more than 1 year.

(SM) short period of moderate malnutrition; $n=13$ : These patients had malnutrition (BMI $-1.45 \pm 0.50$ S.D.s) and secondary amenorrhea for $<1$ year.

$(R)$ recovered from $\mathrm{AN} ; n=15$ : This group was formed of adolescents that had previously suffered AN and had a BMI above -1 s.D.s $(-0.31 \pm 0.62)$ and spontaneous recovery of menses at least 6 months prior to beginning the study and no evidence of bulimia.

Anorexic patients received nutritional and psychiatric therapy throughout the study. Patients with prolonged amenorrhea received oral estrogen (ethynilestradiol $30 \mu \mathrm{g} /$ day) and gestagens (gestodene $75 \mu \mathrm{g}$ /day) during the 24 months of the study. A control group (C) of 23 healthy age-matched Spanish girls $(16.3 \pm 2.6$ years with a range of $11.5-20$ years) with normal BMI (8), pubertal development, and regular menstrual cycles was recruited from a public High School. Controls were studied exclusively at baseline, with no further follow-up.

The study was approved by the Subcommittee on Human Subjects of Hospital Infantil Universitario Niño Jesús and an informed consent was obtained from the subjects and their parents.

\section{Methods}

After the baseline study, AN patients were evaluated every 12 months. Medical and menstrual history, as well as a physical examination, was performed at every visit. Menstruating patients were studied during the follicular stage of the menstrual cycle to avoid possible effects of cycle stage on biochemical and hormonal measurements.

The BMI was calculated by dividing total body weight (kg) by the square standing height $\left(\mathrm{m}^{2}\right)$. All anthropometric measurements are expressed as s.D.s for age and sex referred to a normal Spanish population (8).

Fat mass measurements Total and regional fat mass were evaluated at baseline and every 12 months by whole-body dual-energy X-ray absorptiometry (DEXA; QRD-4500W, Hologic Waltham, MA, USA) using a standard procedure. The anatomical regions explored included the trunk, arms, legs, and total body. The in vivo coefficient of variation (CV) using this technique was below 3\% at each specific regional site. This corresponded to a s.D. of $\sim 415 \mathrm{~g}$ for fat mass and $933 \mathrm{~g}$ for fat-free mass.

Results are expressed in kilograms and the percentage of regional fat mass calculated to analyze the differences between central (trunk) and peripheral (extremity) distribution of fat tissue. The percentage of trunk fat (PTF), percentage of extremity fat (PEF), and ratio of trunk fat to extremity fat (RTEF) were calculated using the following formulas: $\mathrm{PTF}=$ total trunk fat/total fat $X$ $100 ; \mathrm{PEF}=($ arms fat + legs fat $) /$ total fat $) \times 100$; and $\mathrm{RTEF}=\mathrm{PTF} / \mathrm{PEF}$.

Biochemical measurements Blood samples were collected after overnight fasting at $0800 \mathrm{~h}$. Serum estradiol and leptin were measured as previously reported $(6,9)$. Insulin and urinary free cortisol levels were determined by RIA (Diagnostic Products Corp., Los Angeles, CA, USA and Abbot respectively). Intra- and inter-assay $\mathrm{CV}$ values were $<10 \%$.

\section{Statistical analysis}

Data were analyzed using SPSS (10.0) software for Windows (MapInfo Corporation, Troy, NY, USA) and the results are expressed as mean \pm s.D. except when specified (Table 1). Normal distribution of each parameter in every study group was assessed by Kolmogorov-Smirnov goodness of fit. Differences between groups were analyzed for each variable by ANOVA. Comparison of baseline measurements and changes over time were made by Student's $t$-test for paired samples. Pearson's correlation coefficient was used to investigate the association between the parameters studied. The level of significance chosen was $P<0.05$.

\section{Results}

\section{Clinical data}

Baseline clinical data are shown in Table 1. There were no significant differences between the groups in chronological or bone age or in mean age at menarche.

During the follow-up period, BMI remained below -1 S.D.s in the PM group. Patients in the SM group showed significant weight recovery after 12 months $(P<0.05)$ normalizing BMI at 24 months. BMI was in the normal range in $\mathrm{R}$ patients at all time points (Fig. 1). After both 12 and 24 months of follow-up, 12 patients did not experience weight recovery; 3 from the PM group and 9 from the SM group. Menstruation appeared spontaneously in $9(69.2 \%)$ girls in the SM group by 12 months, while the rest remained amenorrheic at 24 months. 
Table 1 Clinical features at baseline.

\begin{tabular}{lcccc}
\hline & \multicolumn{3}{c}{ Groups } \\
\cline { 2 - 5 } & PM & SM & $R$ & Control \\
\hline Chronological age (CA, yr) & $16.1 \pm 1.4$ & $15.0 \pm 1.5$ & $15.6 \pm 1.3$ & $16.3 \pm 2.6$ \\
CA (median, yr) & 16.1 & 15.3 & 15.5 & 16.4 \\
Bone age (yr) & $15.8 \pm 1.1$ & $14.6 \pm 1.2$ & $15.2 \pm 0.9$ & $16.0 \pm 1.9$ \\
BMl (SDS) & $1.69 \pm 0.63^{*}$ & $-1.50 \pm 0.43^{*}$ & $-0.31 \pm 0.62$ & $0.18 \pm 0.72$ \\
Age at diagnosis (yr) & $13.8 \pm 2.0$ & $14.4 \pm 1.7$ & $13.6 \pm 0.9$ & $12.36 \pm 1.43$ \\
Age at menarche (yr) & $12.7 \pm 1.7$ & $12.5 \pm 1.3$ & $11.9 \pm 1.20$ & \\
Secondary amenorrhea (m) & $27.3 \pm 7.1$ & $8.3 \pm 3.9^{\dagger}$ & $9.9 \pm 3.6^{\dagger}$ & \\
Mean duration of AN (m) & $28.1 \pm 12.2$ & $7.4 \pm 4.2^{\dagger}$ & $23.1 \pm 8.5^{\ddagger}$ & \\
\hline
\end{tabular}

${ }^{\star} P<0.05$ compared with control group; ${ }^{\dagger} P<0.05$ compared with $\mathrm{PM} ;{ }^{\ddagger} P<0.05$ compared with SM. SDS, standard deviation score; yr, years; $\mathrm{m}$, months; BMI, body mass index; AN, anorexia nervosa. Groups: PM, prolonged moderate malnutrition; SM, short period of moderate malnutrition; R, recovered from AN.

\section{Body and regional fat and lean mass}

Body fat mass and regional fat and lean distribution are shown in Table 2. Patients in groups PM and SM had low total, trunk, and extremity fat mass throughout the study $(P<0.05)$ with similar upper and lower extremity fat mass reductions regarding controls and without significant differences between them. The only changes in fat mass observed during the follow-up period were increases in total fat at 12 and 24 months and in extremity fat at 12 months in the SM group $(P<0.05)$. No significant differences were found between patients in the recovered group and controls at any time.

Significant differences in the regional distribution of fat mass were only found in the PM group when compared with controls (Table 2), which consisted in a reduction in the PTF throughout the study $(P<0.05)$. The percentage of fat in the extremities was similar to controls in all $\mathrm{AN}$ patients. The ratio of trunk to extremity $(\mathrm{T} / \mathrm{E})$ fat was reduced exclusively in group PM from baseline to the end of the follow-up period $(P<0.05)$ due to the persistently reduced trunk fat.

No significant difference was seen between the anorexic patients and the control group in the percentage of lean mass in the trunk or extremities, or in the ratio of trunk/extremity lean body mass.

Not every patient that increased total body fat had resumption of menses, although increments in total, trunk, and extremity fat were found in patients recovering menses $(1.24 \pm 1.98,0.36 \pm 0.67$, and $0.87 \pm 1.31 \mathrm{~kg}$ respectively), these differences were not significant compared to non-recovering patients $(-0.15 \pm 3.14,-0.10 \pm 0.92$, and $-0.05 \pm 2.22 \mathrm{~kg}$ respectively) probably due to the limited number of cases for this comparison in both subgroups $(n=9$ versus $n=4$ ).

\section{Biochemical data}

Hormonal data are summarized in Table 3. All anorexic patients, except recovered patients, had reduced estradiol levels when compared with controls $(P<0.05)$. Adrenal androgen and urinary free cortisol (UFC) levels were not significantly different between groups. Serum insulin concentrations were significantly lower than controls only in the PM group $(P<0.05)$.

Leptin levels were lower in PM patients when compared with controls $(P<0.05)$ throughout the study. Patients in the SM group normalized their leptin levels after 24 months. No significant differences were found between the recovered group and control subjects. Significant correlations $(P<0.05)$ between leptin levels and total fat mass were found in controls and all AN groups at baseline $(r=0.50,0.60,0.74$, and 0.75 in $C$, PM, SM, and $R$ groups respectively).

Significant correlations were found between changes in S.D.S.-BMI and estradiol at 12 months $(r=0.70$; $P<0.001)$; trunk fat and leptin and insulin at 12 months $(r=0.74, P<0.001$ and $r=0.42, P<0.05$ respectively). In contrast, these correlations were not found after 24 months of follow-up.

\section{Discussion}

Our results demonstrate that although adolescent patients with AN and moderate malnutrition have low

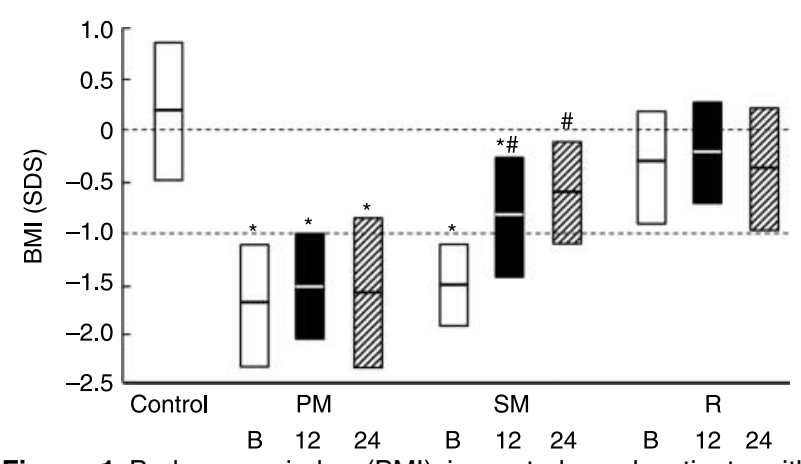

Figure 1 Body mass index (BMI) in controls and patients with prolonged malnutrition (PM), short period of moderate malnutrition (SM), and recovered from AN (R) at baseline (white bar) and after 12 (black bar) and 24 months (hatched bar). The lines in the middle of the bars represent the mean value. ${ }^{*} P<0.05$ compared with control group, ${ }^{\#} P<0.05$ compared with baseline value. 
Table 2 Total and regional fat mass quantification and fat and lean mass distribution.

\begin{tabular}{|c|c|c|c|c|}
\hline & \multicolumn{4}{|c|}{ Groups } \\
\hline & PM & SM & $R$ & Control \\
\hline \multicolumn{5}{|c|}{ Fat mass $(\mathrm{kg})$} \\
\hline Baseline & $8.59 \pm 3.15^{\star}$ & $8.83 \pm 2.38^{\star}$ & $13.26 \pm 3.09$ & \multirow[t]{3}{*}{$14.41 \pm 3.78$} \\
\hline $12 \mathrm{~m}$ & $8.88 \pm 2.21^{*}$ & $10.48 \pm 3.80^{\star \dagger}$ & $14.48 \pm 2.91$ & \\
\hline $24 \mathrm{~m}$ & $7.73 \pm 5.12^{\star}$ & $10.47 \pm 3.00^{\star \dagger}$ & $13.25 \pm 2.88$ & \\
\hline \multicolumn{5}{|c|}{ Trunk fat (kg) } \\
\hline Baseline & $2.88 \pm 1.17^{*}$ & $3.12 \pm 0.97^{\star}$ & $4.75 \pm 1.39$ & \multirow[t]{3}{*}{$5.55 \pm 1.71$} \\
\hline $12 \mathrm{~m}$ & $3.14 \pm 1.26^{*}$ & $3.44 \pm 1.33^{*}$ & $5.29 \pm 1.36$ & \\
\hline $24 \mathrm{~m}$ & $2.53 \pm 1.95^{\star}$ & $3.61 \pm 1.22^{*}$ & $4.77 \pm 1.24$ & \\
\hline \multicolumn{5}{|c|}{ Extremity fat $(\mathrm{kg})$} \\
\hline Baseline & $5.01 \pm 2.07^{\star}$ & $4.96 \pm 1.60^{\star}$ & $7.81 \pm 1.78$ & \multirow{3}{*}{$8.12 \pm 2.20$} \\
\hline $12 \mathrm{~m}$ & $5.31 \pm 1.57^{\star}$ & $6.32 \pm 2.57^{\star \dagger}$ & $8.45 \pm 1.69$ & \\
\hline $24 \mathrm{~m}$ & $4.50 \pm 3.19^{*}$ & $6.01 \pm 1.96^{\star}$ & $7.73 \pm 1.82$ & \\
\hline \multicolumn{5}{|l|}{ PTF (\%) } \\
\hline Baseline & $32.59 \pm 3.09^{*}$ & $35.54 \pm 5.40$ & $35.43 \pm 3.35$ & \multirow[t]{2}{*}{$38.24 \pm 3.98$} \\
\hline $12 \mathrm{~m}$ & $32.39 \pm 3.35^{\star}$ & $33.51 \pm 4.08$ & $36.17 \pm 3.84$ & \\
\hline $24 \mathrm{~m}$ & $30.00 \pm 8.01^{*}$ & $34.26 \pm 3.99$ & $35.86 \pm 34.02$ & \\
\hline \multicolumn{5}{|l|}{ PEF (\%) } \\
\hline Baseline & $56.90 \pm 7.12$ & $55.12 \pm 7.67$ & $59.03 \pm 2.79$ & \multirow[t]{2}{*}{$56.37 \pm 3.73$} \\
\hline $12 \mathrm{~m}$ & $58.93 \pm 5.77$ & $58.70 \pm 6.91$ & $58.54 \pm 3.39$ & \\
\hline $24 \mathrm{~m}$ & $56.47 \pm 7.30$ & $58.16 \pm 7.45$ & $58.33 \pm 4.11$ & \\
\hline \multicolumn{5}{|l|}{ RTEF } \\
\hline Baseline & $0.55 \pm 0.08^{*}$ & $0.62 \pm 0.15$ & $0.60 \pm 0.08$ & \multirow[t]{3}{*}{$0.68 \pm 0.12$} \\
\hline $12 \mathrm{~m}$ & $0.53 \pm 0.07^{*}$ & $0.59 \pm 0.15$ & $0.62 \pm 0.10$ & \\
\hline $24 \mathrm{~m}$ & $0.54 \pm 0.16^{\star}$ & $0.59 \pm 0.11$ & $0.62 \pm 0.11$ & \\
\hline \multicolumn{5}{|l|}{ PTL (\%) } \\
\hline Baseline & $47.11 \pm 3.93$ & $49.79 \pm 1.72$ & $48.90 \pm 1.32$ & \multirow[t]{2}{*}{$49.52 \pm 1.67$} \\
\hline $12 \mathrm{~m}$ & $49.97 \pm 2.18$ & $50.41 \pm 1.90$ & $49.27 \pm 1.99$ & \\
\hline $24 \mathrm{~m}$ & $48.43 \pm 1.70$ & $49.83 \pm 1.98$ & $48.41 \pm 1.88$ & \\
\hline \multicolumn{5}{|l|}{ PEL(\%) } \\
\hline Baseline & $40.98 \pm 4.42$ & $42.02 \pm 1.58$ & $42.86 \pm 1.40$ & \multirow[t]{3}{*}{$42.82 \pm 1.72$} \\
\hline $12 \mathrm{~m}$ & $42.09 \pm 1.68$ & $41.45 \pm 2.06$ & $42.88 \pm 2.04$ & \\
\hline $24 \mathrm{~m}$ & $44.02 \pm 1.61$ & $42.20 \pm 1.93$ & $44.41 \pm 2.10$ & \\
\hline
\end{tabular}

${ }^{\star} P<0.05$ compared with control group; ${ }^{\dagger} P<0.05$ compared with baseline. PTF, percentage of trunk fat; PEF, percentage of extremity fat; RTEF, ratio of trunk fat to extremity fat; PTL, percentage of trunk lean; PEL, percentage of extremity lean; $m$, months. Groups: PM, prolonged moderate malnutrition; SM, short period of moderate malnutrition; R, recovered from AN.

body fat content, only those patients with prolonged malnutrition have an altered fat distribution when compared with controls, with a greater fat loss from the trunk. Weight recovery was associated with an adequately distributed fat mass acquisition, with no changes in regional fat percentages or in RTEF. Leptin levels did not change after initial BMI recovery, but normalized after BMI reached control values.

DEXA is currently the method of choice to measure body composition although, as every technique, its

Table 3 Hormonal data.

\begin{tabular}{|c|c|c|c|c|}
\hline & \multicolumn{4}{|c|}{ Groups } \\
\hline & PM & SM & $R$ & Control \\
\hline \multicolumn{5}{|c|}{ Estradiol (pg/ml) } \\
\hline Basal & $21.85 \pm 14.9^{*}$ & $25.22 \pm 19.2^{*}$ & $51.27 \pm 18.3$ & \multirow[t]{3}{*}{$94.26 \pm 16.6$} \\
\hline $12 \mathrm{~m}$ & $15.97 \pm 14.9^{*}$ & $25.25 \pm 19.3^{*}$ & $66.87 \pm 30.8$ & \\
\hline $24 \mathrm{~m}$ & $12.99 \pm 10.8^{*}$ & $30.60 \pm 16.6^{*}$ & $61.60 \pm 12.4$ & \\
\hline \multicolumn{5}{|c|}{ Insulin $(\mu \mathrm{U} / \mathrm{ml})$} \\
\hline Basal & $5.30 \pm 2.2^{*}$ & $8.33 \pm 5.0^{\dagger}$ & $7.71 \pm 3.0$ & \multirow[t]{3}{*}{$10.05 \pm 2.9$} \\
\hline $12 \mathrm{~m}$ & $4.70 \pm 1.8^{*}$ & $8.24 \pm 5.3^{\dagger}$ & $8.29 \pm 3.1$ & \\
\hline $24 \mathrm{~m}$ & $4.79 \pm 2.6^{\star}$ & $7.42 \pm 4.3$ & $6.69 \pm 1.8$ & \\
\hline \multicolumn{5}{|c|}{ Leptin (ng/ml) } \\
\hline Basal & $4.33 \pm 1.9^{*}$ & $8.38 \pm 4.8^{* \dagger}$ & $10.20 \pm 3.8$ & \multirow[t]{3}{*}{$15.25 \pm 8.1$} \\
\hline $12 \mathrm{~m}$ & $4.87 \pm 1.4^{\star}$ & $8.03 \pm 3.4^{\star \dagger}$ & $11.38 \pm 3.0$ & \\
\hline $24 \mathrm{~m}$ & $5.10 \pm 3.2^{*}$ & $9.05 \pm 2.8^{\dagger}$ & $11.01 \pm 3.7$ & \\
\hline
\end{tabular}

${ }^{\star} P<0.05$ compared with control group; ${ }^{\dagger} P<0.05$ compared with $\mathrm{PM} ; \mathrm{m}$, months. Groups: $\mathrm{PM}$, prolonged moderate malnutrition; SM, short period of moderate malnutrition; $R$, recovered from AN. 
accuracy in determining the total fat content and its distribution is limited. The RTEF is used to evaluate body fat distribution and previous authors have reported that AN adolescents with a BMI below -2 s.D.s have a low RTEF due to a greater reduction in trunk fat mass $(10,11)$. Our results are in agreement with these studies, but we found that adolescents with a short period of malnutrition maintained a normal fat distribution pattern despite having a reduced BMI. Hence, the time of evolution of AN may also be a determinant of alterations in fat distribution regarding controls, although we were not able to find differences between PM and SM groups neither in fat distribution nor in BMI-S.D.s at baseline, may be influenced by the limited size of the groups. This may also explain why we did not see a difference in lean body mass distribution as reported by Misra et al., with similar bone age between groups (12).

During nutritional therapy, weight gain is quicker in the early recovery period with a tendency to lay down more fat in following phases (13). In AN patients under nutritional treatment, fat mass is reported to represent from 21 to $77 \%$ of the total weight gain (14). We observed an increase in fat mass only during the first 12 months in short-term moderately malnourished patients, consistent with the higher caloric intake requirement at the beginning of treatment in order to increase weight quickly. In spite of BMI normalization at 24 months, patients with short-term malnutrition did not normalize fat mass, what was not found in recovered patients; thus, fat mass may require a more prolonged recuperation period than BMI.

Some authors have postulated that fat mass distribution after refeeding could differ between adults and adolescents with AN. In adults, weight gain is accompanied by a significant increase in trunk fat with central adiposity $(4,15,16)$. This has also been reported in partially weight recovered adolescents (11), suggesting that central adiposity may develop in these girls. In contrast, other authors have shown a normal fat distribution pattern after weight gain in adolescent patients with similar characteristics (17). In our study, patients who had short-term moderate malnutrition achieved a normal BMI and a partial restoration of fat mass, while patients in the recovered group sustained a normal BMI and total fat mass throughout the 2-year period. Neither of these groups showed an increase in trunk adiposity. These results support the hypothesis that weight recovery results in a tendency toward normalization of body fat mass and not of increased central adiposity.

It has been postulated that rapid weight gain could be associated with an abnormal fat mass distribution, whereas it is uncertain whether therapies leading to slow and sustained weight gain could be more beneficial (4). We have observed that from the initial BMI restoration in patients with short-term moderate malnutrition to the complete fat mass recovery and long-term maintenance in the recovered group, body fat distribution remains unchanged. These findings differ from those by Misra et al. (11) who reported that shortterm weight gain in AN adolescents was associated with an increase in trunk fat and subsequently in RTEF, although they did not exceed control values. This difference may be due to the lack of initial affection of RTEF in our patients in the SM group. Our long-term follow-up of partially and fully recovered patients suggests that neither partial nor complete fat mass restoration induces the development of trunk adiposity in moderately affected AN adolescents.

The pathophysiological implications of changes in regional fat distribution in $\mathrm{AN}$ patients remain unknown. Some authors have postulated that in adult AN females, the marked hypercortisolemia could influence central fat distribution (18). However, we found normal UFC levels in AN adolescents and perhaps this could be involved in the prevention of central adiposity, nevertheless, this issue must be considered cautiously taking into account domiciliary urine collection and lack of correction for creatinine excretion. In addition, altered growth hormone (GH) secretion dynamics in AN (19) could be involved in fat distribution; unfortunately, we were not able to study $\mathrm{GH}$ secretion in these patients.

Serum leptin levels were reduced in AN patients with malnutrition and amenorrhea as previously reported $(6,20)$. Moreover, we found that changes in leptin levels in SM group were not temporally correlated to the BMI increase in SM patients and that a prolonged period of normal body weight was necessary to return leptin levels to normal (6). Interestingly, despite having similar BMI, total, and regional fat content, patients in PM group showed lower leptin levels than SM group at baseline.

Plasma insulin concentrations are also proportional to the amount of corporal fat and a relationship with leptin levels has been reported (21). Once again, despite having similar BMI, total, and regional fat content, patients in PM group showed lower insulin levels than SM group at baseline. These changes could be informing about a more severe impairment of patients in PM group, due to the longer duration of malnutrition.

A critical minimum amount of body weight and fat is necessary for resumption of menses in malnourished states $(22,23)$. Indeed, due to the influence of leptin on the hypothalamic-pituitary-gonadal axis, the increase in leptin levels after fat mass recovery is one of the determinants of menses resumption $(24,25)$. Others authors have reported that menstruation recovery occurs at $\sim 90 \%$ of adequate weight for height and age, independently of fat mass (26). By the end of our study, a high percentage of patients from the SM group and those from recovered group showed spontaneous recuperation of menses. Short-term moderately malnourished adolescents had a partial increase in fat mass at 12 months of therapy and leptin levels normalized at 
24 months, whereas patients in the recovered group had normal fat content and leptin levels. These findings support the hypothesis that a critical fat mass is necessary for the recuperation of menstrual function and that leptin plays a relevant role in gonadal function (27). The persistence of amenorrhea in AN patients who reach a normal BMI could be due to the coexistence of estradiol and leptin deficiencies and the maintenance of specific psychopathological impairments (28). Moreover, it is possible that although the patient's BMI is normalized, they may not have reached their metabolic optimum (29).

In conclusion, AN adolescents with PM have an abnormal regional fat distribution due to a greater loss of trunk fat. Long-term therapies with slow weight recovery lead to fat mass restoration without impairment of regional distribution. Finally, the evaluation of body fat appears to be a more accurate indicator of nutritional status than BMI in these patients.

\section{Acknowledgements}

The authors wish to thank Dr Julie A Chowen for the critical review of the manuscript. Gabriel Ángel MartosMoreno is supported by the Fondo de Investigación Sanitaria (FIS CM05/00100). This work was held by CIBER (CB 06/03/0022) and the Fundación Endocrinología y Nutrición.

\section{References}

1 Hudson JI, Hiripi E, Pope HG Jr \& Kessler RC. The prevalence and correlates of eating disorders in the national comorbidity survey replication. Biological Psychiatry 200761 348-358.

2 Mazess RB, Barden HS \& Ohlrich ES. Skeletal and body composition effects of anorexia nervosa. American Journal of Clinical Nutrition 199052 438-441.

3 Zamboni M, Armellini F, Turcato E, Todisco P, Gallagher D, Dalle Grave R, Heymsfield S \& Bosello O. Body fat distribution before and after weight gain in anorexia nervosa. International Journal of Obesity and Related Metabolic Disorders 199721 33-36.

4 Mayer L, Walsh BT, Pierson RN Jr, Heymsfield SB, Gallagher D, Wang J, Parides MK, Leibel RL, Warren MP, Killory E \& Glasofer D. Body fat redistribution after weight gain in women with anorexia nervosa. American Journal of Clinical Nutrition 200581 1286-1291.

5 Meier U \& Gressner AM. Endocrine regulation of energy metabolism: review of pathobiochemical and clinical chemical aspects of leptin, ghelin, adiponectin and resistin. Clinical Chemistry 200450 1511-1525.

6 Argente J, Barrios V, Chowen JA, Sinha MK \& Considine RV. Leptin plasma levels in healthy Spanish children and adolescents, children with obesity, and adolescents with anorexia nervosa and bulimia nervosa. Journal of Pediatrics 1997131 833-838.

7 American Psychiatric Association. Diagnostic and Statistical Manual of Mental Disorders, Text Revised (DSM-IV-TR) version 4. Washington, DC: American Psychiatric Press, 2000.

8 Hernández M, Castellet J, Narvaiza JL, Rincón JM, Ruiz E, Sánchez E, Sobradillo B \& Zurimendi A. In Curvas y Tablas de Crecimiento. Instituto de Investigación sobre Crecimiento y Desarrollo. Fundación Faustino Orbegozo. Madrid: Editorial Garsi, 1988.
9 Argente J, Hervás F, Barrios V, Pozo J, Muñoz M, González S, PérezMérida MC \& Hernández M. Valores normales de glucurónido de $3 \alpha$ androstanodiol, proteína transportadora de los esteroides sexuales, testosterona total, testosterona libre, estradiol, $\Delta$-4-androstendiona, sulfato de dehidroepiandrosterona y 17-hidroxiprogesterona en la infancia, adolescencia y época adulta en ambos sexos: modificaciones en función de la edad y del sexo. Endocrinología 1993 40 23-32.

10 Kerruish KP, O’Connor J, Humphries IRJ, Kohn MR, Clarke SD, Briody JN, Thomson ES, Wright KA, Gaskin KJ \& Baur LA. Body composition in adolescents with anorexia nervosa. American Journal of Clinical Nutrition 200275 31-37.

11 Misra M, Soyka L, Miller K, Grinspoon S, Levitsky L \& Klibanski A. Regional body composition in adolescents with anorexia nervosa and changes with weight recovery. American Journal of Clinical Nutrition 200377 1361-1367.

12 Misra M, Miller KK, Almazan C, Worley M, Herzog DB \& Klibanski A. Hormonal determinants of regional body composition in adolescent girls with anorexia nervosa and controls. Journal of Clinical Endocrinology and Metabolism 2005 90 2580-2587.

13 Standard KL, Wills VG \& Waterlow JC. Indirect indicators of muscle mass in malnourished infants. American Journal of Clinical Nutrition 195933 2686-2693.

14 Polito A, Cuzzolaro M, Raguzzini A, Censi L \& Ferro-Luzzi A. Body composition changes in anorexia nervosa. European Journal of Clinical Nutrition 199852 655-662.

15 Iketani T, Kiriike N, Nagata T \& Yamagami S. Altered body fat distribution after recovery of weight in patients with anorexia nervosa. International Journal of Eating Disorders 199926 275-282.

16 Grinspoon S, Thomas L, Miller K, Pitts S, Herzog D \& Klibanski A. Changes in regional fat redistribution and the effects of estrogen during spontaneous weight gain in women with anorexia nervosa. American Journal of Clinical Nutrition 200173 865-869.

17 Kooh SW, Noriega E, Leslie K, Muller C \& Harrison JE. Bone mass and soft tissue composition in adolescents with anorexia nervosa. Bone $199619181-188$.

18 Epel ES, McEwen B, Seeman T, Matthews K, Castellazzo G, Brownell KD, Bell J \& Ickovics JR. Stress and body shape: stress-induced cortisol secretion is consistently greater among women with central fat. Psychosomatic Medicine 200062 623-632.

19 Argente J, Caballo N, Barrios V, Muñoz MT, Pozo J, Chowen JA, Morandé G \& Hernández M. Multiple endocrine abnormalities of the growth hormone and insulin-like growth factor axis in patients with anorexia nervosa: effect of short- and long-term weight recuperation. Journal of Clinical Endocrinology and Metabolism 199782 2084-2092.

20 Nakai Y, Hamagaki S, Kato S, Seino Y, Takagi R \& Kurimoto F. Leptin in women with eating disorders. Metabolism 199948 217-220.

21 Bagdade JD, Bierman EL \& Porte DJ. The significance of basal insulin levels in the evaluation of the insulin response to glucose in diabetic and nondiabetic subjects. Journal of Clinical Investigation 196746 1549-1557.

22 Swenne I. Weight requirements for return of menstruations in teenage girls with eating disorders, weight loss and secondary amenorrhoea. Acta Paediatrica 200493 1449-1455.

23 Misra M, Prabhakaran R, Miller KK, Tsai P, Lin A, Lee N, Herzog DB \& Klibanski A. Role of cortisol in menstrual recovery in adolescent girls with anorexia nervosa. Pediatric Research 200659 598-603.

24 Holtkamp K, Mika C, Grzella I, Heer M, Pak H, Hebebrand J \& Herpertz-Dahlmann B. Reproductive function during weight gain in anorexia nervosa. Leptin represents a metabolic gate to gonadotropin secretion. Journal of Neural Transmission 2003110 427-435.

25 Misra M, Miller KK, Almazan C, Ramaswamy K, Aggarwal A, Herzog DB, Neubauer G, Breu J \& Klibanski A. Hormonal and body composition predictors of soluble leptin receptor, leptin, and free 
leptin index in adolescent girls with anorexia nervosa and controls and relation to insulin sensitivity. Journal of Clinical Endocrinology and Metabolism 200489 3486-3495.

26 Golden NH, Jacobson MS, Schebendach J, Solanto MV, Hertz SM \& Shenker R. Resumption of menses in anorexia nervosa. Archives of Pediatrics and Adolescent Medicine 1997151 16-21.

27 Rogol AD. Editorial: leptin and puberty. Journal of Clinical Endocrinology and Metabolism $1998 \mathbf{8 3}$ 1089-1090.

28 Brambilla F, Monteleone P, Bortolotti F, Grave RD, Todisco P, Favaro A, Santonastaso P, Ramacciotti C, Paoli R \& Maj M.
Persistent amenorrhoea in weight-recovered anorexics: psychological and biological aspects. Psychiatry Research 2003118 249-257.

29 Crisp AH \& Stonehill E. Relation between aspects of nutritional disturbance and menstrual activity in primary anorexia nervosa. BMJ 19713 149-151.

Received 11 July 2007

Accepted 12 July 2007 